\title{
Hot topics in ventilator-associated pneumonia
}

Ventilator-associated pneumonia (VAP) remains an important clinical problem, being associated with significant morbidity and mortality. In this special issue of Annals of Translational Medicine, novel aspects regarding definition, diagnosis, epidemiology, and treatment of VAP are discussed by experts in this field.

The new classification of ventilator associated events (VAE) proposed by the Centers of Disease Control and prevention enhances the understanding of mechanical ventilation complications, including both infection-related and non-infective events. Dr. Rello and colleagues discuss the advantages and disadvantages of ventilator-associated respiratory infections (VARI) classification and the new CDC VAE-paradigm. They report that VARI and VAE classifications are focused on different targets, and suggest that a more comprehensive and complete strategy should combine both algorithms with clinical experience.

Better understanding of pathophysiology of VAP could be useful for future studies aiming at improving prevention and treatment of this infection. Dr. Makris and colleagues discuss data suggesting that under intra-abdominal hypertension conditions, bacterial translocation might be an additional responsible mechanism for VAP in critically ill patients that merits further investigation in the future.

Oropharyngeal colonization and VAP mechanisms are tightly linked. A significant within-population variation in oropharyngeal colonization has been described, with its composition being dependent from patients' severity. Dr. Messika and colleagues discuss the importance of oropharyngeal colonization in critically ill patients, and recent data against the use of chlorhexidine in these patients.

An accurate and early diagnosis of VAP is crucial to reach the optimal balance between prompt appropriate antibiotic treatment and multi-drug resistance prevention. Dr. Bouhemad and colleagues discuss recent data on the interest of routine use of lung ultrasound as an attractive alternative to bedside chest radiography in diagnosing VAP.

Subglottic secretion drainage (SSD) is one of the recommended strategies to prevent VAP with a high level of evidence, especially regarding early-onset pneumonia. Dr. Lacherade and colleagues report that all meta-analyses found that the use of subglottic secretion drainage reduces VAP occurrence with a relative risk reduction of $45 \%$, and that SSD reduces the duration of mechanical ventilation but without beneficial effect on ICU or hospital mortality. They discuss why this measure is not widely used in critically ill patients.

Endotracheal aspirate (ETA) surveillance cultures have been used to predict the microorganisms responsible for VAP in intensive care unit patients. Dr. Hraiech and colleagues report that a large number of studies, with heterogeneous designs and variable results, have questioned the possibility of predicting, by regular ETA cultures after the 48th hour of mechanical ventilation, the microbiology of VAP and therefore of determining the adequate antibiotic therapy to limit the overprescription of broad-spectrum molecules when following guidelines.

The multimodal approach for VAP prevention has been shown to be a successful strategy in reducing VAP rates in many intensive care units in some countries. The simultaneous application of several measures or "bundles" to reduce VAP rates has achieved a higher impact than the progressive implementation of the individual interventions. Dr. Alvarez-Lerma and colleagues discuss the selection of the individual recommendations of the bundle, education of care workers (HCW) in the culture of patient safety, audit of compliance with the recommendations, commitment of the hospital management to support implementation, nomination and empowerment of local leaders of the projects in ICUs, both physicians and nurses, and the continuous collection of VAP episodes.

Extracorporeal membrane oxygenation (ECMO) provides a circulatory and/or respiratory assistance in case of refractory cardiogenic shock or acute respiratory distress syndrome (ARDS). Due to their extreme critical illness, these patients usually require prolonged mechanical ventilation, which is an inherent risk of VAP. Dr. Schmidt and colleagues diagnosis and treatment of VAP in ECMO patients.

The worldwide spreading of extended spectrum beta-lactamase producing enterobacteriaceae (ESBL-PE) represents a major problem encountered more and more frequently in ICU. Whereas, previous carriage is the major risk factors associated with VAP related to ESBL-PE, among carriers, only $5 \%$ to $20 \%$ will develop a VAP related to ESBL-PE. Dr. Zahar and 
colleagues discuss the recent findings on VAP related to ESBL-PE, the relationship between colonization and VAP, and the best therapeutic options.

As management of VAP requires adequate and timely antibiotic administration, global emergence of antimicrobial resistance poses serious challenges over our ability to maintain this axiom. Dr. Garyphallia and colleagues discuss newly approved and forthcoming agents for the treatment of VAP related to resistant Gram-negative bacilli, such as ceftolozane/ tazobactam, ceftazidime/avibactam, cefiderocol, aztreonam/avibactam, murepavadin, and plazomicin.

\section{Acknowledgements}

None.

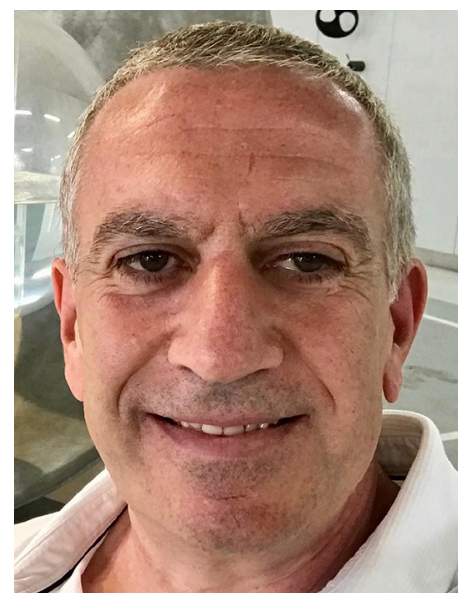

Saad Nseir

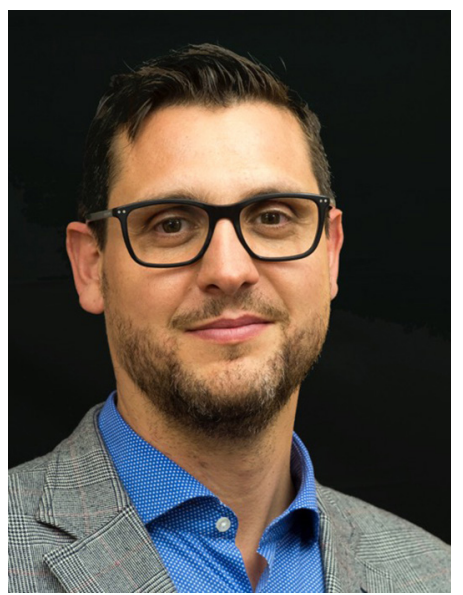

Ignacio Martin-Loeches 